Open Access

\title{
Electro-acupuncture for treatment of knee pain from osteoarthritis and the possible endocrinology changes: a study protocol for a randomized controlled trial
}

Javier Mata ${ }^{1 *}$, Sandra Cabrera ${ }^{2}$, Pilar Sanchís ${ }^{3}$, Pedro Valentí ${ }^{1}$, Patricia Hernández ${ }^{1}$, Regina Fortuny ${ }^{4}$, Serafin Lirola ${ }^{5}$ and Jose Luis Aguilar ${ }^{1}$

\begin{abstract}
Background: Osteoarthritis of the knee is a major cause of disability among adults. Electro-acupuncture is considered a potentially useful treatment for osteoarthritis. The purpose of this study is to assess the efficacy of electro-acupuncture on pain control, pain perception, plasma cortisol and beta-endorphin levels, patient-perceived quality of life, and pain medication use in patients with chronic knee pain.

Methods/design: This study is a placebo-controlled, randomized, double-blind, parallel design trial. One hundred sixty out-patients who are more than 50 years old and who have osteoarthritis of the knee will be recruited from the island of Mallorca, Spain. Each participant will be randomly placed into one of two groups: (sham) electro-acupuncture non-insertion technique and real electro-acupuncture.

Acupuncture treatments will be the Traditional Chinese Medicine type. The patients will be evaluated after a period of 1 month (with two weekly sessions), 3 months (with one monthly session), 6 months (with one session every 45 days), and 1 year later with follow-up sessions at the end of the study (with one session every 2 months).

The primary outcomes will be based on the observed changes from the baseline of the visual analogue scale (VAS) and the Western Ontario and McMaster Universities Osteoarthritis Index (WOMAC) for pain measured at 12 weeks after the end of treatment.

Also to be included in the study are the possible changes in the secondary efficacy variables from baseline as assessed by the Short Form 36 version 2 health survey (patient-perceived quality of life), patient plasma cortisol and beta-endorphin levels at the different treatment stages, the Goldberg Anxiety and Depression Scale, pain medication use, functional capacity and stiffness (WOMAC subscales), and a VAS. These variables will be assessed at 1 month, 3 months, 6 months, and 1 year after study commencement.
\end{abstract}

Discussion: The findings from this study will help to determine whether electro-acupuncture is effective for chronic knee pain management in older people and whether electro-acupuncture can deliver results for the improvement of pain relief, stiffness, and disability. The study will therefore be a major step toward understanding the roles of the hypothalamic-pituitary-adrenal axis and the endogenous opioid system in the effectiveness of electro-acupuncture for chronic pain.

Trial registration: ClinicalTrials.gov identifier NCT02299713 (11 Nov. 2014).

Keywords: Electro-acupuncture, Knee osteoarthritis, Chronic pain, Osteoarthritis, Beta-endorphin, Non-puncturing needle, Cortisol

\footnotetext{
* Correspondence: jmata@hsll.es

'Anaesthesiology and Clinic Pain Department, Son Llàtzer Hospital, Ctra. de

Manacor kilómetro 4, 07198 Palma de Mallorca, Spain

Full list of author information is available at the end of the article
}

\section{Biomed Central}

(c) 2015 Mata et al. This is an Open Access article distributed under the terms of the Creative Commons Attribution License (http://creativecommons.org/licenses/by/4.0), which permits unrestricted use, distribution, and reproduction in any medium, provided the original work is properly credited. The Creative Commons Public Domain Dedication waiver (http:// creativecommons.org/publicdomain/zero/1.0/) applies to the data made available in this article, unless otherwise stated. 


\section{Background}

Osteoarthritis (OA) is the most common form of arthritis $[1,2]$. Its prevalence is greater among women than men $[3,4]$. Several factors contribute to the risk of OA, including age, gender, genetics, behavioral influences, obesity, injury, and reduced muscular strength [5]. Significant consequences of OA are activity limitations, reduced participation in work and social activities, and mental distress.

The most recent clinical guidelines based upon evidence from the UK National Institute of Clinical Excellence [6] and the Osteoarthritis Research International (OARSI) suggest that the treatment for OA be multidisciplinary in nature and consider non-pharmacological treatment (such as education, aerobic and resistance exercises, and weight loss) as well as pharmacological treatment options (paracetamol) when an additional treatment is required. In an OARSI systematic review, five of the eight guidelines considered acupuncture to be one of the 12 possible non-pharmacological therapy modalities for OA [7].

Acupuncture has been demonstrated to be a safe treatment with a low risk of serious side effects [8-12]. OA guidelines $[13,14]$ emphasize treatment safety as an important issue, especially when the therapeutic agents for elderly patients with knee OA and comorbidities are concurrent drugs that could have serious, life-threatening interactions.

Acupuncture could be used to explain the roles that the hypothalamic-pituitary-adrenal (HPA) axis and the endogenous opioid (EO) system play as important mediators of pain, the stress response, and other stimuli [15]. Recent neuroimaging studies by positron emission tomography have shown that the relationship between the neurochemical imbalance in the EO system [16] and the long-term pain relief produced by acupuncture practice has the opposite results of those observed with placebo (sham) acupuncture and that only acupuncture exerts a long-term effect through the EO system [17].

The effect of acupuncture on the HPA system is far from clear. Although cortisol release is one of the consequences of HPA system activation, different studies have shown opposite responses of cortisol release in response to acupuncture. One mediating factor may be the presence or absence of anxiety and depression, which are known to predict the onset and severity of chronic pain [18].

Although many studies have demonstrated the efficacy of acupuncture in the treatment of OA-associated knee pain [19], only one study [20] demonstrates the effectiveness of electro-acupuncture (EA) for pain reduction that includes the indicative reduced cortisol levels and elevated blood beta-endorphins within the same patient group. It would be of great interest to determine whether the lack of these kinds of results could be masked by interfering factors such as anxiety and depression.
When acupuncture was compared with some control groups (waiting list and control groups with active treatment), the positive effects reached the threshold of clinical relevance. Moreover, the only non-pharmacological therapeutic treatment for OA shown to reduce pain and improve physical function was exercise, and this treatment either closely met or exceeded the threshold for clinical relevance [21].

Although many studies show the efficacy of acupuncture for OA pain, the lack of a common methodology for the trial design complicates the possibility of comparing the respective results in order to determine treatment efficacy.

In this work, we have collected the following methodological suggestions for acupuncture studies that would minimize the design biases: the number of patients recruited to achieve the significant differences, treatment number, long-term treatment $[22,23]$, recruitment blinding method, EA use [24, 25], assessment of patient perceptions about the type of technique used before and after treatment [26], the objective and subjective assessment of the technique, and finally the use of sham with no needle penetration (a non-penetrating sham control). Thus, our aim is to apply these methods to verify the effectiveness of acupuncture for OA pain. Furthermore, our placebo use would permit the validation of the hypothesis.

Additionally, the current economic reality that dictates the need to develop favorable cost-benefit therapies for both patients and the health-care system should not be overlooked, and acupuncture is a cost-effective therapy for the treatment of knee OA [27].

Finally, a great deal of evidence indicates that various possible physiological mechanisms explain how acupuncture may provide pain relief, and these indicate that further research is needed to clarify the interrelationship and regulation of these mechanisms.

Although cortisol release is one of the consequences of HPA system activation, some studies have shown responses opposite to the cortisol release produced by acupuncture practice. Normal individuals show opposite cortisol responses to those with chronic pain conditions. This suggests that if the HPA axis contributes to the efficacy of acupuncture, its role is more complex than simply deactivating this system. Rather the contribution may depend on the population being studied. One of the objectives of this study is to provide more data to elucidate possible mechanisms of action and to show correlations between reductions in pain and changes in hormonal markers of HPA axis function. This will aid in establishing whether changes in HPA axis are causal or merely epiphenomenal in treatment efficacy.

This following study is a project to assess the effectiveness of EA for the treatment of common pathologies that involve chronic OA pain in knee. Additionally, it 
will evaluate pain perception, plasma endorphin and cortisol levels, patient quality of life, and the need for pharmacological drugs regardless of whether EA is applied.

\section{Hypothesis}

\section{Conceptual hypothesis}

Patients who receive EA will have, to some degree, better pain control than non-treated patients.

\section{Operative hypothesis}

Patients who receive EA will have a measured basal pain perception that is three points less on the visual analogue scale (VAS) after EA treatment in comparison with non-treated patients.

\section{Statistical hypothesis}

Practice of EA will result in a statistically significant control of pain compared with the pain levels of nontreated patients.

\section{Methods/design Trial design}

This study proposes a randomized, double-blind, placebo-controlled, pre- and post-test, parallel design clinical trial which conforms to the CONSORT (Consolidated Standards of Reporting Trials) [28] and STRICTA (Standards for Reporting Interventions in Clinical Trials of Acupuncture) guidelines [29] for acupuncture studies (Fig. 1).

\section{Participants}

Participants will be selected from a population with knee chronic pain caused by OA ( $>3$ months in duration), from the communities of the island of Mallorca (Balearic Islands, Spain). These participants will be recruited in the outpatient clinics of the island's hospitals and advertisements in local newspapers and through the hospital website (http://www.hsll.es). Advertisements for patient recruitment will not specify acupuncture as one of the treatments being investigated or offered. Doctors from the trauma services of local hospitals will be invited to screen patients. The clinical trial will be performed by the Pain Unit at Son Llàtzer Hospital, Palma de Mallorca.

A total of 160 patients will be necessary (80 subjects for each treatment group) to detect differences of at least three points in the pain perception assessment according to VAS (scale of 0 to 10). Accepted values will be for an alpha risk of $1 \%$ and beta risk of less than $5 \%$ in a bilateral contrast as well as a value of 4 for the standard deviation (size effect of 0.75). It is assumed that $20 \%$ of the trial patients will be lost to attrition. Patients will be included in the study by case-consecutive, non- probability sampling after responding to a recruitment visit to the Pain Clinic; then if they sign an informed consent form, they will be placed randomly into one of the treatment groups [30].

\section{Inclusion criteria}

Eligibility requirements will include the following: male and female patients who are more than 50 years old and who have knee OA diagnosed according to the American College of Rheumatology classification criteria (with at least one osteophyte in the tibiofemoral joint and with a Kellgren-Lawrence grade of more than 2), who have had a history of moderate or greater knee pain of more than 3 months' duration and have reported moderate or greater, clinically significant, knee pain during most days of the last month before the interview, and who have given their consent to participate in the study. Patients will be selected among those who are not on the waiting list for total knee arthroplasty by the traumatology unit of our hospital.

\section{Exclusion criteria}

Patients with any of the following will be excluded from the study: a history of any secondary OA associated with any systemic arthropathy (i.e., rheumatoid arthritis or gouty arthritis); any knee treatment with steroids, methotrexate, or azathioprine; recent traumatisms caused by acupunctural insertion; a history of bleeding disorders or any disease related to acupuncture contraindications; a history of any knee injection in the previous 6 months (e.g., cortisone and hyaluronic acid); an acupuncture treatment history; or a history of morphine or morphine derivative use.

\section{Removal criteria}

Patients will be removed by voluntary removal or by failure to consecutively attend two or more EA sessions.

\section{Variables}

\section{Main variables}

The primary outcomes will be the changes from the baseline of the VAS and the Western Ontario and McMaster Universities Osteoarthritis Index (WOMAC) for pain at the completion of treatment at 12 weeks.

\section{Secondary variables}

Secondary variables to be considered are the following: the change in the secondary efficacy variables from the baseline of the Short Form 36 version 2 health survey (SF-36v2) (patient-perceived quality of life), the change in plasma cortisol and beta-endorphin levels, the scores for the Goldberg Anxiety and Depression Scale (GADS), 


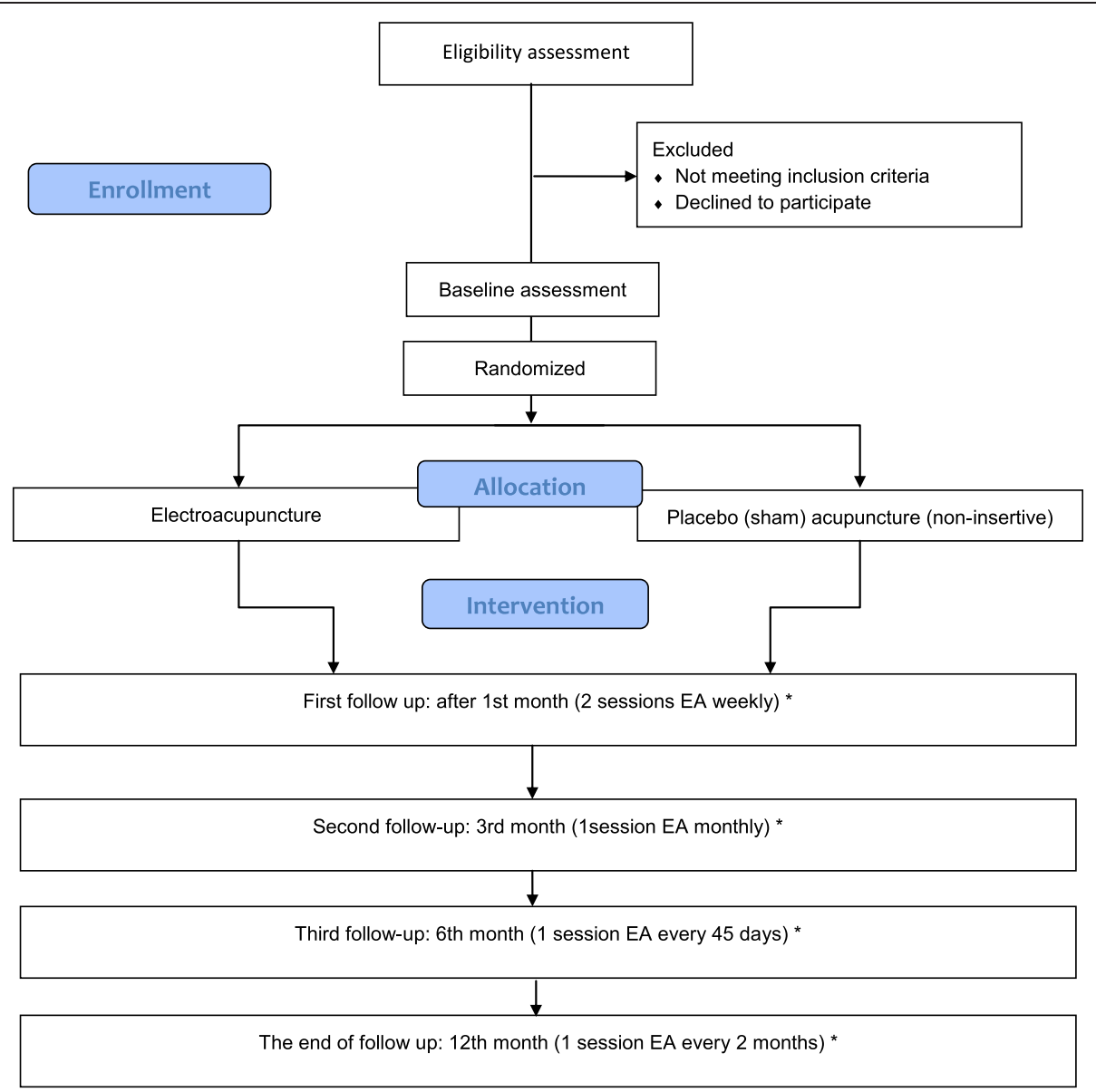

Fig. 1 Trial flow. The study trial flow is described, indicating the patient selection process, treatment, and follow-up. *Visual analogue scale, Western Ontario and McMaster Universities Osteoarthritis Index, Short Form 36 version 2 health survey, blood sample collection, Goldberg Anxiety and Depression Scale, and medication use are measured on each follow-up visit. EA, Electro-acupuncture

changes in pain medication use, changes in functional capacity and stiffness (WOMAC subscales), and VAS scores measured at 1 month, 3 months, 6 months, and 1 year after study commencement.

\section{Procedure}

Figure 1 describes the trial protocol. The eligibility of prospective participants will be determined by a researcher who is not involved in the assessment or treatment of the participants. Ethical approval was obtained from the Balearic Islands Human Research Ethics Committee in July 2013 (CEI-IB, \#IB 2077/13 PI).

Upon arrival at the Clinic for Pain Treatment, recruited patients will be informed once again about the trial objectives, and any questions or doubts that the patient might still have with respect to the study will be resolved. Patients will then be informed that they are testing a new EA technique for knee pain treatment. Placebo or sham acupuncture (SA) will not be mentioned to the patient during the interview or referred to in the informed consent form. Subjects will be placed into one of two study groups (with a strict $50 \%$ probability), and to reinforce and encourage treatment follow-up, patients will be told that the effects are to be perceived over the medium- to long-term time frame. Patients will be reassessed to ensure that they still comply with the selection criteria and that they have not acquired any new criteria for exclusion. Participants will provide informed consent in the first baseline visit (Table 1).

\section{Baseline visit}

The researcher will re-collect clinical data of patients, including age, sex, and body mass index. In the baseline interview, the participants will not yet be assigned to a treatment group.

- Pain perception assessment will be based on (1) the VAS score and (2) the WOMAC scale pain score. The VAS is a continuous scale made up of a $10-\mathrm{cm}$, unmarked, horizontal line, upon which the subject 
Table 1 Planned visit schedule for treatment and follow-up, indicating visit number and frequency

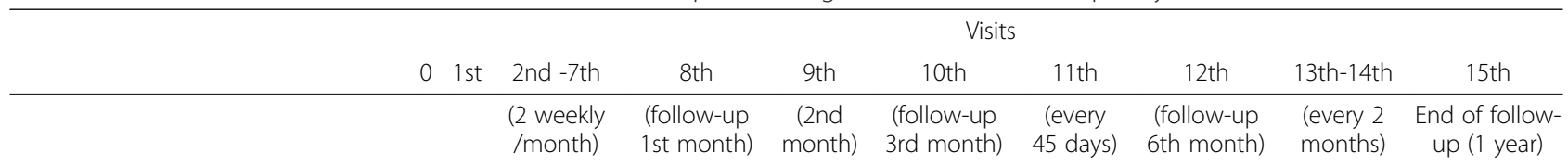

Patients

\begin{tabular}{|c|c|c|c|c|c|c|c|c|c|}
\hline $\begin{array}{l}\text { Patient's evaluation and collection of } \\
\text { the relevant data }\end{array}$ & $\sqrt{ }$ & & & & & & & & \\
\hline Inclusion/Exclusion criteria & $\sqrt{ }$ & & & & & & & & \\
\hline $\begin{array}{l}\text { Explanation of the objectives of the } \\
\text { procedure and how it works }\end{array}$ & $\sqrt{ }$ & & & & & & & & \\
\hline Informed consent & $\sqrt{ }$ & & & & & & & & \\
\hline Randomization and blinding & $\sqrt{ }$ & & & & & & & & \\
\hline Expectation of acupuncture & & & $\sqrt{ }$ & & $\sqrt{ }$ & & $\sqrt{ }$ & & $\sqrt{ }$ \\
\hline Treatment & & & & & & & & & \\
\hline Electro-acupuncture (EA) & $\sqrt{ }$ & $\sqrt{ }$ & $\sqrt{ }$ & $\sqrt{ }$ & $\sqrt{ }$ & $\sqrt{ }$ & $\sqrt{ }$ & $\sqrt{ }$ & $\sqrt{ }$ \\
\hline Sham acupuncture (SA) & $\sqrt{ }$ & $\sqrt{ }$ & $\sqrt{ }$ & $\sqrt{ }$ & $\sqrt{ }$ & $\sqrt{ }$ & $\sqrt{ }$ & $\sqrt{ }$ & $\sqrt{ }$ \\
\hline Outcomes & & & & & & & & & \\
\hline Visual analogue scale (VAS) & $\sqrt{ }$ & & $\sqrt{ }$ & & $\sqrt{ }$ & & $\sqrt{ }$ & & $\sqrt{ }$ \\
\hline $\begin{array}{l}\text { McMaster Universities Osteoarthritis } \\
\text { Index (WOMAC) }\end{array}$ & $\sqrt{ }$ & & $\sqrt{ }$ & & $\sqrt{ }$ & & $\sqrt{ }$ & & $\sqrt{ }$ \\
\hline Medication use & $\sqrt{ }$ & & $\sqrt{ }$ & & $\sqrt{ }$ & & $\sqrt{ }$ & & $\sqrt{ }$ \\
\hline $\begin{array}{l}\text { Goldberg Anxiety and Depression } \\
\text { Scale }\end{array}$ & $\sqrt{ }$ & & $\sqrt{ }$ & & $\sqrt{ }$ & & $\sqrt{ }$ & & $\sqrt{ }$ \\
\hline Short Form Health Survey (SF-36v2) & $\sqrt{ }$ & & $\sqrt{ }$ & & $\sqrt{ }$ & & $\sqrt{ }$ & & $\sqrt{ }$ \\
\hline Blood sample collection & $\sqrt{ }$ & & $\sqrt{ }$ & & $\sqrt{ }$ & & $\sqrt{ }$ & & $\sqrt{ }$ \\
\hline Trial evaluation & & & & & & & & & \\
\hline Adverse event & & & $\sqrt{ }$ & & $\sqrt{ }$ & & $\sqrt{ }$ & & $\sqrt{ }$ \\
\hline Outcome assessment & & & & & & & & & $\sqrt{ }$ \\
\hline Reasons of drop-outs or withdrawals & & & $\sqrt{ }$ & & $\sqrt{ }$ & & $\sqrt{ }$ & & $\sqrt{ }$ \\
\hline Satisfaction and expectations Survey & & & & & & & & & $\sqrt{ }$ \\
\hline
\end{tabular}

makes a hash mark depending on the amount of pain felt; a mark on the far left of the line indicates "no pain" (score of 0 ), and a mark to the far right qualifies as "pain as bad as it could be" or "unbearable pain" (score of 100). The WOMAC scale is used to evaluate the condition of patients with OA of the knee and the hip and includes pain, stiffness, and physical functioning of the joints. This scale measures five items for pain (with a score range of 0-20), two for stiffness (score range of $0-8$ ), and 17 for functional limitation (score range of 0-68). The physical functioning questions cover everyday activities. These scales will be used separately and will not be summed. Patients will respond orally to the five levels with the following criteria: "none" $=0$, "a bit" = 1, "quite a bit" = 2, "a lot" = 3, and "very much" $=4$. If two or more questions are left unanswered, the scale will be declared invalid. If the patient does not respond to one question, a mean will be taken from the results of the other questions. The range will be $0-98$.

- Analgesic medicine use. This questionnaire will have four questions developed according the EUROHIS (European Health Interview Survey) recommendations in order to harmonize the information regarding medicine use [31]. Subjects will be asked (1) about the prescription medicine their general practitioner may have prescribed for them ("Have you taken any pain medicine prescribed by your general practitioner?") as well as any medication not prescribed by their general practitioner ("Have you taken any pain medicine not prescribed by your general practitioner") and (2) whether or not their prescribed and non-prescribed pain medication use has increased or decreased.

- The degree of functionality and stiffness according to the WOMAC scale. There are two questions for stiffness (scale of 0-8) and 17 questions for 
functional limitation (score range of 0-68). Each question will be answered orally with the following criteria: "none" = 0 , "a bit" = 1, "quite a bit" = 2, "a lot" $=3$, and "very much" $=4$. If two or more questions are unanswered, the scale scores will be declared invalid and not counted.

- Anxiety/depression level according to the GADS. This consists of two subscales (of nine questions for each scale): an anxiety scale (1-9) and a depression scale (10-18). The first questions for each scale (1-4 and 10-13) are preconditioned questions to determine whether the patient should attempt to answer the rest of the questions of the two subscales. Effectively, at least two questions must be answered affirmatively in the first scale (1-4) for the patient to continue; in the second scale, one answer is sufficient for questions $10-13$ for the patient to continue with the rest of the questions. This study will use the Spanish version validated by Montón et al. [32].

- Degree of quality of life. This will be assessed according to the SF-36v2 questionnaire and is compounded by 36 items, which yield an 8-scale profile of functional health and well-being scores as well as psychometrically based physical and mental health summary measures and a preference-based health utility index. The Spanish version of the SF-36 questionnaire by Ware and Sherbourne [33] has been correctly translated and standardized and has been given the Spanish title of Cuestionario de Salud SF-36 [34].

The first baseline plasmatic sample will be extracted from the antecubital vein to evaluate beta-endorphin and cortisol levels, and the sampling time will be between 9 and $14 \mathrm{~h}$. To avoid any diurnal variation in plasmatic hormonal levels, subjects will always be assigned the same hour for the extractions. Five blood samples (5 $\mathrm{ml}$ each) from each patient will be taken under aseptic conditions from the antecubital vein; one extraction will required before the treatment, one at 1 month after the start of treatment, one at 3 months, one at 6 months, and one at the end of treatment. Except for the first pretreatment sample, extractions will take place $5 \mathrm{~min}$ after finishing the acupuncture treatment. Once extracted, blood will be placed in a refrigerated tube with EDTA (ethylenediaminetetraacetic acid) anticoagulant and centrifuged immediately. Plasma will be extracted with a micropipette and divided in two equal fractions (one for the cortisol and one for the beta-endorphin analysis). Samples will be stored at $-70{ }^{\circ} \mathrm{C}$ until the analysis. The baseline interview treatment will have no assigned sampling time and will occur on the first day, when the subject is assigned to his or her respective treatment group.

\section{First day of treatment Randomization}

Each eligible patient will be randomly assigned to one of two groups: "A" and "B". A random number list generated by SPSS statistical software version 18.0 (IBM Corporation, Armonk, NY, USA) (balanced for each six cases per study branch) will be used for the allocation to each group. Researchers from the statistical center will send the randomized list in a numbered, sealed, and opaque envelope to the researcher responsible for participant recruitment and group assignment.

\section{Blinding}

The patient codes of the double-blind study will be placed in numbered, sealed, and opaque envelopes. Researchers, personnel performing the interviews, statisticians, and participants will be blinded to patient allocation. Study groups will consist of the following, although patients will have no knowledge into which study branch they have been placed: the experimental group (A) receiving EA or the control group (B) receiving SA.

\section{Intervention}

The treatment will be applied in eight sessions (two per week) for 1 month. Group A patients will receive the EA treatment, and group $B$ will receive the SA treatment. Both groups will receive two weekly sessions for 4 weeks, then one session (each month) for the first 3 months, one session (each 45 days) until 6 months, and finally one session every 2 months until finalizing the study after a year's time.

Treatments will be performed by the same acupuncturist, an anesthesiology specialist who has studied Traditional Chinese Medicine (TCM) at the University of Chinese Medicine, Beijing, China, holds a master's degree in acupuncture from the University of León, Spain, and has more than 8 years of clinical experience in therapeutic pain management by acupuncture.

Experimental group (group A) EA will be performed for a 20-30 min treatment session, and the patients will be semi-seated comfortably on a stretcher. A $0.30 \times 30$ $\mathrm{mm}$ needle will be used and will make a $10-30 \mathrm{~mm}$ insertion into the skin, depending on the patient's constitution. Needle insertion points will be selected according to the TCM meridian theory for knee pain treatment. Upon insertion, EA will be applied by a biphasic pulse generator apparatus at a frequency of $3 \mathrm{~Hz}$ and at the maximum tolerable intensity. Table 2 shows the local and distal points: St 34 (Liáng Qiū), localized 2 cun proximal to the upper lateral border of the patella, in a groove of the vastus lateralis muscle; St 35 ( $D \dot{u} B \hat{\imath})$, in the depression inferior to the patella and lateral to the patellar ligament; St 36 (Zú Sān Lù), one fingerbreadth 
Table 2 Framework of acupuncture points that should be selected when administering acupuncture treatments

\begin{tabular}{|c|c|c|}
\hline Location & Acupuncture points & Description \\
\hline \multicolumn{3}{|c|}{ Local points } \\
\hline & St 34 (Liáng Qiū) & 2 cun proximal to the upper lateral border of the patella, in a groove of the vastus lateralis muscle. \\
\hline & St 35 (Dú Bi) & In the depression inferior to the patella and lateral to the patellar ligament. \\
\hline & St 36 (Zú Sān Lì) & One fingerbreadth lateral to the anterior crest of the tibia, on the tibialis anterior muscle. \\
\hline & Lv 8 (Qū Quán) & $\begin{array}{l}\text { Proximal to the medial end of the popliteal crease, in a depression anterior to the tendons of the } \\
\text { semitendinosus and semimembranosus muscles. }\end{array}$ \\
\hline & Sp 9 (Yìn Líng Quán) & $\begin{array}{l}\text { In a depression distal to the medial condyle of the tibia, at the junction of the shaft and the medial } \\
\text { condyle. }\end{array}$ \\
\hline & Sp 10 (Xuè Hăi) & $\begin{array}{l}2 \text { cun proximal and slightly medial to the medial superior border of the patella, in a depression on } \\
\text { the vastus medialis muscle. }\end{array}$ \\
\hline & Ki 10 (Yìn Gŭ) & $\begin{array}{l}\text { At the medial end of the popliteal crease, between the tendons of the semimembranosus and } \\
\text { semitendinosus muscles, on the level of the knee joint space. }\end{array}$ \\
\hline & M-Le 16 (Xi Yan) & Inferior to the patella, medial and lateral to the patellar ligament. \\
\hline & M-Le 27 (Hè Ding) & In the center of the upper border of the patella. \\
\hline
\end{tabular}

Distal point

St 44 (Nèi Tíng) Between the second and third toes, proximal to the interdigital fold.

lateral to the anterior crest of the tibia, on the tibialis anterior muscle; Lv 8 (Qü Quán), proximal to the medial end of the popliteal crease, in a depression anterior to the tendons of the semitendinosus and semimembranosus muscles; Sp 9 (Yìn Líng Quán), in a depression distal to the medial condyle of the tibia, at the junction of the shaft and the medial condyle; Sp 10 (Xuè Hăi), 2 cun proximal and slightly medial to the medial superior border of the patella, in a depression on the vastus medialis muscle; Ki 10 (Yin Gü), at the medial end of the popliteal crease, between the tendons of the semimembranosus and semitendinosus muscles, on the level of the knee joint space; M-Le 16 (Xi Yan), inferior to the patella, medial and lateral to the patellar ligament; and M-Le 27 (Hè Ding), in the center of the upper border of the patella. As a distal point, St 44 (Nèi Tíng), between the second and third toes, proximal to the interdigital fold, will be used.

The needles will be inserted through the same guide tube that will be used for the control group. The objective is for control patients to perceive the De-Qi sensation, which is like a tingling or numbness on the specific points punctured. Subsequently, EA will be used between Xiyan points and also to the points that the patient recalls as the area of pain. If both knees are affected, the same acupuncture points will be applied.

To ensure that the treatment procedures for the treatment and the control groups are as similar as possible, we will tape two guide tubes to two sham points at the abdominal area, approximately $3 \mathrm{~cm}$ lateral to and slightly above the umbilicus bilaterally. Immediately after, we will affix a pair of needles by adhesive tape to the surface at these same points though without needle insertion. This site was chosen for two reasons. First, these sites were at two meridians that are theoretically irrelevant to knee pain. Second, to facilitate blinding, we wanted to provide the participants of both groups the opportunity to be able to feel a sensation similar to the actual insertion of the needle.

Control group (group B) A valid method of placebo acupuncture with non-insertion of the needle will be used for the control group [35]. Two needles will be inserted into the sham points of the abdominal area in the same position used for group A, approximately $3 \mathrm{~cm}$ lateral to and slightly above the umbilicus bilaterally, and then two pieces of adhesive tape will be immediately taped next to the needles. In addition, a mock plastic needle guide tube will be placed on the surface of each of the nine true points in the leg so that some discernable sensation will be produced; then a needle will be immediately applied without needle insertion to the dermal surface at each point and will be retained at this position for 20-30 min. These plastic guide tubes will be applied in the same manner as for group A in order to produce the same perceptible sensation.

The control group will receive the same session and schedule as group A. This will include the same active needle placements, although needle insertion will not occur at these nine points (local and distal points). Not only will the accessories of the EA equipment be connected in the same point in group A without electric stimulation, a mock transelectrical stimulation unit (which emits a "timer" sound and has a blinking light) will be attached to the sham needles at the knee.

To facilitate patient blinding for both groups, a screen will be placed below the abdomen to prevent participants from actually observing true or sham procedures 
at the knee area but at the same time will permit them to see the procedure that is being given in the abdominal area. It is important to state that this type of intervention will need some time for the results to be noted, and a thorough follow-up is necessary to avoid dropouts from both groups.

\section{Next several visits for treatment (two sessions per week until the completion of eight sessions)}

The same treatment will be repeated according to group. In the last session, blood samples will be drawn $5 \mathrm{~min}$ after the treatment completion, at the same time of day as the previous extraction.

\section{At 1 month's time}

The interviewers will repeat the assessments for pain level, analgesic consumption, and the type of treatment that the patient believes he or she is receiving as well as the assessment by the WOMAC scale and the GADS. The patient will once again answer the quality-of-life questionnaire (SF-36v2). Once the forms have been completed, treatment will be administered to the patient.

\section{Subsequent visits}

At 3 and 6 months after treatment start and at the final visit, the questionnaires again will be filled out. The interviewers will also note EA-related adverse effects or complications observed either by the participants or by the acupuncturist throughout the duration of the treatment. Treatments will be performed each month up until the third month, and after this latter treatment session, with just one treatment every 45 days until the sixth month. Finally, a treatment will occur every 2 months until the study is completed at the end of 1 year. Blood tests will be performed again on the third month, on the sixth month, and at the end of treatment period.

\section{Study end}

At study completion, a questionnaire will be given that includes questions related to patient satisfaction with the treatment received, the type of treatment patients believe they have received, and their expectations for improvement.

\section{Statistical analysis}

The results will be analyzed by using the SPSS statistical software version 18.0. A descriptive analysis of the collected data will be obtained by using the mean, the standard deviation, and the maximum and minimum values for the quantitative variables that follow a normal distribution. For variables without a normal distribution (degree of pain, plasma levels, and quality of life, among others), the median, the 25th and 75th percentiles, and maximum and minimum values will be calculated.
Qualitative variables will be expressed as frequencies and percentages. Differences shall be detected by chisquare or Fisher tests for categorical variables and Student $t$ or Mann-Whitney tests for the resultant variables.

\section{Discussion}

The aim of the study is to measure the effectiveness of EA in alleviating OA knee pain. Effectiveness indicators should be the relief of pain, stiffness and functional disability of the knee, the improvement of quality of live and a reduction in medication use.

The principal challenge expected with this study is the difficulty of effectively blinding SA application in a manner that is believable or credible for the patient. For this reason, we will avoid using the terms "sham acupuncture" or "placebo" in the information letter and consent form. The attempt to blind the process will be done by explaining to the patient that a new pain treatment will be assessed and compared with other techniques (infiltrations and analgesic stimulation) and that the selected patients must have no history of acupuncture treatment. The literature reports that the non-penetrating SA method has been widely used and has also been validated for avoiding skin receptor stimulation in SA [36]. Disclosure of a patient's group affiliation will be avoided so that they will not be able to share information among themselves or compare treatments. The only person who will have full knowledge of the applied treatment will be the acupuncturist, although he will remain blind to the rest of the details of the study.

A satisfaction questionnaire will be completed at the end of the study to assess whether the patients believe they have or have not received treatment. To avoid introducing bias that could be caused by the interference of the circadian rhythm in the beta-endorphin and cortisol levels, patients will always be scheduled for blood sampling at the same hour.

As has been shown, it would seem clear that the treatment of knee OA must be a multidisciplinary approach, as there is currently no treatment alone that may be considered beneficial in absolute terms.

Although there are studies showing the efficacy of acupuncture for the treatment of OA pain, the lack of a common methodology to apply the treatment design complicates the comparison of the results between studies.

In this article, we combine all of the methodological suggestions for acupuncture studies, attempting to minimize the biases that may result from study design. We intend to make a comparison with a placebo that would allow the hypothesis to be validated.

We must not forget the current economic circumstances that drive us to look for therapies that are costbeneficial for both the patient and the health system. In 
this direction, there are studies that suggest acupuncture as a cost-effective therapy for the treatment of knee OA.

Finally, sufficient evidence about the different physiological mechanisms would encourage further research on how acupuncture can relieve pain. However, the effect of acupuncture on the HPA system is not clear, and therefore we intend to provide more information that would clarify its function.

\section{Trial status}

The trial is currently in the recruitment phase. Participant recruitment started in January 2015 and is expected to end in December 2015.

\begin{abstract}
Abbreviations
EA: Electro-acupuncture; EO: Endogenous opioid system; GADS: Goldberg Anxiety and Depression Scale; Group A: Experimental group; Group B: Control group; HPA: Hypothalamic-pituitary-adrenal; OA: Osteoarthritis; OARSI: Osteoarthritis Research International; SA: Sham acupuncture; SF-36v2: Short Form 36 version 2 health survey; TCM: Traditional Chinese Medicine; VAS: Visual analogue scale; WOMAC: Western Ontario and McMaster Universities Osteoarthritis Index.
\end{abstract}

\section{Competing interests}

The authors declare that they have no competing interests.

\section{Authors' contributions}

$J M$ is leading the trial coordination and helped to conceive the project, develop the protocol, obtain project funding, design the acupuncture program, and write the first and final drafts of the manuscript. SC helped to conceive the project and design the statistical analysis and will help to perform the sample size calculations. PS helped to develop the protocol, obtain project funding, design the statistical analysis, and write the first draft of the manuscript and will help to perform the sample size calculations. PV helped to design the acupuncture program. RF will be directing the blood sample analyses. SL will head participant recruitment. PH will recruit and screen the participants and perform data entry. JLA helped to write the first and final drafts of the manuscript. All authors participated in the trial design, provided feedback on drafts of this article, and read and approved the final manuscript.

\section{Acknowledgments}

This trial is being funded by our own research funds. We wish to acknowledge the valuable efforts of Jose Antonio Ribas, Ainhoa Reta, Maria del Mar Moya, Margarita Buades, Miguel Nolla, Magdalena Molina and Catalina Tortell, who are team members and belong to the Pain Clinic staff. We would like to express our sincere appreciation for the excellent work of John Sibole and Ileana Martinez Cabrera, language editors who have made significant revisions to the manuscript, editing style, and corrections.

\section{Author details \\ 'Anaesthesiology and Clinic Pain Department, Son Llàtzer Hospital, Ctra. de Manacor kilómetro 4, 07198 Palma de Mallorca, Spain. ${ }^{2}$ Catalan Institute of Oncology, Germans Trias I Pujol Hospital, Ctra. Del Canyet s/n., 08916 Badalona, Spain. ${ }^{3}$ Research Unit, Son Llàtzer Hospital, Ctra. de Manacor kilómetro 4, 07198 Palma de Mallorca, Spain. ${ }^{4}$ Clinical and Haematology Analysis Laboratory, Son Llàtzer Hospital, Ctra. de Manacor kilómetro 4, 07198 Palma de Mallorca, Spain. ${ }^{5}$ Traumatology Department, Son Llàtzer Hospital, Ctra. de Manacor kilómetro 4, 07198 Palma de Mallorca, Spain.}

Received: 18 December 2014 Accepted: 20 May 2015

Published online: 03 June 2015

\section{References}

1. Lawrence RC, Helmick CG, Arnett FC, Deyo RA, Felson DT, Giannini EH, et al. Estimates of the prevalence of arthritis and selected musculoskeletal disorders in the United States. Arthritis Rheum. 1998;41:778-99.
2. Felson DT, Lawrence RC, Dieppe PA, Hirsch R, Helmick CG, Jordan JM, et al. Osteoarthritis: new insights. Part 1: the disease and its risk factors. Ann Intern Med. 2000;133:635-46.

3. Bijlsma JW, Berenbaum F, Lafeber FP. Osteoarthritis: an update with relevance for clinical practice. Lancet. 2011;377:2115-26.

4. Zhang Y, Jordan JM. Epidemiology of osteoarthritis. Clin Geriatr Med. 2010;26:355-69.

5. Ausiello JC, Stafford RS. Trends in medication use for osteoarthritis treatment. J Rheumatol. 2002;29:999-1005.

6. National Collaborating Centre for Chronic Conditions. Osteoarthritis: national clinical guideline for care and management in adults. London: Royal College of Physicians; 2008.

7. Zhang FW, Moskowitz RW, Nuki G, Abramson S, Altman RD, Arden N. OARSI recommendations for the management of hip and knee osteoarthritis, Part II: OARSI evidence-based, expert consensus guidelines. Osteoarthritis Cartilage. 2008;16:137-62

8. MacPherson $\mathrm{H}$, Thomas $\mathrm{K}$, Walters S, Fitter M. The York acupuncture safety study: prospective survey of 34000 treatments by traditional acupuncturists. BMJ. 2001;323:486-7.

9. White A, Hayhoe S, Hart A, Ernst E. Adverse events following acupuncture: prospective survey of 32000 consultations with doctors and physiotherapists. BMJ. 2001;323:485-6.

10. Cherkin DC, Sherman KJ, Deyo RA, Shekelle PG. A review of the evidence for the effectiveness, safety, and cost of acupuncture, massage therapy, and spinal manipulation for back pain. Ann Intern Med. 2003;138:898-906.

11. Lao L, Hamilton GR, Fu J, Berman BM. Is acupuncture safe? A systematic review of case reports. Altern Ther Health Med. 2003;9:72-83.

12. Melchart D, Weidenhammer W, Streng A, Reitmayr S, Hoppe A, Ernst E, et al. Prospective investigation of adverse effects of acupuncture in 97733 patients. Arch Intern Med. 2004;164:104-5.

13. American College of Rheumatology Subcommittee on Osteoarthritis Guidelines. Recommendations for the medical management of osteoarthritis of the hip and knee: 2000 update. Arthritis Rheum. 2000;43:1905-15.

14. Jordan K, Arden N, Doherty M, Bannwarth B, Bijlsma J, Dieppe P, et al. EULAR Recommendations 2003: an evidence based approach to the management of knee osteoarthritis: Report of a Task Force of the Standing Committee for International Clinical Studies Including Therapeutic Trials (ESCISIT). Ann Rheum Dis. 2003;62:1145-55.

15. Herman JP, Figueiredo H, Mueller NK, Ulrich-Lai Y, Ostrander MM, Choi DC, et al. Central mechanisms of stress integration: hierarchical circuitry controlling hypothalamo-pituitary-adrenocortical responsiveness. Front Neuroendocrinol. 2003;24:151-80.

16. Harris RE, Zubieta JK, Scott DJ, Napadow V, Gracely RH, Clauw DJ. Traditional Chinese acupuncture and placebo (sham) acupuncture are differentiated by their effects on [mu]-opioid receptors (MORs). Neuroimage. 2009;47:1077-85.

17. Zubieta JK, Stohler CS. Neurobiological mechanisms of placebo responses. Ann N Y Acad Sci. 2009;1156:198-210.

18. Jones GT, Harkness EF, Nahit ES, McBeth J, Silman AJ, Macfarlane GJ. Predicting the onset of knee pain: results from a 2-year prospective study of new workers. Ann Rheum Dis. 2007;66:400-6.

19. Selfe TK, Taylor AG. Acupuncture and osteoarthritis of the knee: a review of randomized, controlled trials. Fam Community Health. 2008;31:247-54.

20. Ahsin S, Saleem S, Bhatti AM, lles RK, Aslam M. Clinical and endocrinological changes after electro-acupuncture treatment in patients with osteoarthritis of the knee. Pain. 2009;147:60-6.

21. Fransen M, McConnell S, Bell M. Exercise for osteoarthritis of the hip or knee. Cochrane Database Syst Rev. 2003;3, CD004286.

22. Berman BM, Lao L, Langenberg P, Lee WL, Gilpin AM, Hochberg MC. Effectiveness of acupuncture as adjunctive therapy in osteoarthritis of the knee: a randomized, controlled trial. Ann Intern Med. 2004;141:901-10.

23. Witt C, Brinkhaus B, Jena S, Linde K, Streng A, Wagenpfeil S, et al. Acupuncture in patients with osteoarthritis of the knee: a randomised trial. Lancet. 2005;366:136-43.

24. Sangdee C, Teekachunhatean S, Sananpanich K, Sugandhavesa N, Chiewchantanakit S, Pojchamarnwiputh S, et al. Electroacupuncture versus diclofenac in symptomatic treatment of osteoarthritis of the knee: a randomized controlled trial. BMC Complement Altern Med. 2002;2:3.

25. Vas J, Méndez C, Perea-Milla E, Vega E, Panadero MD, León JM, et al. Acupuncture as a complementary therapy to the pharmacological 
treatment of osteoarthritis of the knee: randomised controlled trial. BMJ. 2004;329:1216

26. Moerman $D E$, Jonas WB. Deconstructing the placebo effect and finding the meaning response. Ann Intern Med. 2002;136:471-6.

27. Whitehurst DG, Bryan S, Hay EM, Thomas E, Young J, Foster NE. Cost-effectiveness of acupuncture care as an adjunct to exercise-based physical therapy for osteoarthritis of the knee. Phys Ther. 2011;91:630-41.

28. Schulz KF, Altman DG, Moher D. CONSORT 2010 statement: updated guidelines for reporting parallel group randomised trials. BMJ. 2010;340:c332.

29. MacPherson H, Altman DG, Hammerschlag R, Li Y, Wu T, White A, et al. Revised Standards for Reporting Interventions in Clinical Trials of Acupuncture (STRICTA): extending the CONSORT statement. Acupunct Med. 2010;28:83-93.

30. Shuster JJ. Practical handbook of sample size guidelines clinical trials MAC. Boca Raton, FL: CRC Press; 1992.

31. EUROHIS: Developing Common Instruments for Health Surveys. A. Nosikov and C. Gudex (Eds.) Amsterdam: IOS Press; 2003

32. Montón C, Pérez Echeverría MJ, Campos R, García Campayo J, Lobo A. Anxiety scales and Goldberg's depression: an efficient interview guide for the detection of psychologic distress. Aten Primaria. 1993;12:345-9.

33. Ware JE Jr, Sherbourne CD. The MOS 36-item short-form health survey (SF-36). I Conceptual Framework Item Selection Med Care. 1992;30:473-83.

34. Alonso J, Prieto L, Antó JM. The Spanish version of the SF-36 Health Survey (the SF-36 health questionnaire): an instrument for measuring clinical results. Med Clin (Barc). 1995;104:771-6.

35. Lao L, Bergman S, Hamilton GR, Langenberg P, Berman B. Evaluation of acupuncture for pain control after oral surgery: a placebo-controlled trial. Arch Otolaryngol Head Neck Surg. 1999;125:567-72.

36. Gong $X$, Pan Z, Shen $Y$, Wang S. Blinding effect of non-penetrating sham needle in the randomized controlled trials of acupuncture: a systematic review. J Acupunct Tuina Sci. 2014;12:8-11. doi:10.1007/s11726-014-0738-1.

\section{Submit your next manuscript to BioMed Central and take full advantage of:}

- Convenient online submission

- Thorough peer review

- No space constraints or color figure charges

- Immediate publication on acceptance

- Inclusion in PubMed, CAS, Scopus and Google Scholar

- Research which is freely available for redistribution 